\title{
Activeapp: A Smartphone-based Movement Activity Monitoring System for Healthcare
}

\author{
Godwin Ogbuabor \\ Department of Computer Science \\ Michael Okpara University of Agriculture, Umudike \\ Abia State, Nigeria
}

\author{
F. N. Ugwoke \\ Department of Computer Science \\ Michael Okpara University of Agriculture, Umudike \\ Abia State, Nigeria
}

\begin{abstract}
Participating in physical activities is necessary for people of all ages. It positions individuals in a state of fitness, thereby enhancing the quality of life. Physical inactivity, which can result in obesity and overweight will not only affect the quality of life, but equally bring financial burden to the government and relevant individuals. The manufacturers of mobile devices such as smartphones recently incorporated some sensors such as accelerometer, step counter, step detector. Their high computational power, low cost, and small size make it possible for people to carry such accessories always. The embedded sensors can be used to track human steps in real time. In this work, we present a prototype mobile application using step counter sensor embedded in smartphones to track daily steps of users carrying mobile phones. The app serves as a motivating factor for engaging in physical activities such as taking stairs instead of taking the lift, walking instead of driving to work, and also running to increase the rate of heart beat. Experimental evaluation of the mobile application shows that the app could be used to track and record daily steps of people carrying mobile phones effectively.
\end{abstract}

\section{Keywords}

Smartphone, Healthcare, Mobile Application, Activity Monitoring

\section{INTRODUCTION}

The healthcare industry is rapidly responding to the use of mobile phones for effective and efficient healthcare delivery. Mobile application is transforming healthcare the same way it has changed the way people live, work and interact with each other. Smallman[5] stated that wellbeing and health applications are made up of approximately $40 \%$ of recently developed mobile applications. The manufacturers of mobile devices such as smartphones recently incorporated many powerful sensors such as accelerometer, step sensor, GPS, temperature and blood pressure sensors [6]. These sensors can be utilized to develop mobile applications that can potentially assist to manage people's health [4]. Their high computational power, low cost, and small size make it possible for people to carry them always [8]. There is no doubt that mobile healthcare applications will massively facilitate a future in which patients will be given the appropriate tools to manage their health [4]. Chronic diseases such as obesity, diabetes and cardiovascular diseases could potentially managed by automatic monitoring of patientls daily activities [3]. These patients are usually required to follow a definite active exercise routine such as walking and running as part of their treatment [8]. Physical activity has good effect against chronic diseases, it helps to stimulate the immune system and reduce the risk of cardiovascular diseases [4]. Participating in physical activity is necessary for people of all ages [13]. It positions individual in a state of fitness, thereby, enhancing the quality of' life. Research shows that handling depression with exercise can serve as medication to people suffering from mental health problems [13]. Clinical studies have also shown that increasing the number of steps one take in a day can assist people to stay active [2]. It is clear that to reduce the global epidemic of obesity and overweight, people should be encouraged to participate in daily exercises by embarking in some physical activities like walking, running and cycling.

Report from World Health Organization (WHO) shows that, the major cause of overweight and obesity is lack of physical exercise to balance energy between consumed calories and expended calories [1]. Regular physical activities reduces depression, anxiety and promote sense of well-being [1] Physical inactivity which can result in obesity and overweight will not only affect the quality of life, but equally bring financial burden to the government and individuals. The aim of this work is therefore to implement mobile based movement activity monitoring system (ActiveApp) using step counter sensor embedded in mobile phones, to monitor and manage individual health. We are mainly interested in motivating people to engage in physical activities such as taking the stairs instead of taking lift, walking instead of driving to work, and also to run to increase the heartbeat rate.

The rest of the work is organized as follows: section two presents related works by other researchers, section three discusses the system architecture and implementation, while section four presents the experimental evaluation. Finally, section five concludes the work.

\section{RELATED WORK}

Report from World Health Organization (WHO) shows that, the major cause of overweight and obesity is lack of physical exercise needed to balance energy between consumed calories and expended calories). Regular physical activities reduce depression, anxiety and promote sense of well-being [1]. Studies have shown that people can monitor and manage their health using mobile applications with the help of sensors embedded in the phones. Maitland et al. [10] presented a prototype mobile application to record daily physical activities of users using fluctuation of the signal strength of GSM cells. The mobile app was evaluated with three different groups to determine if the app could enhance users' awareness of their activity level and the possibility of motivating them to be more active. They pointed out that this method is a reliable way of sensing activity and has advantages over popular methods of using accelerometer sensor in that there is no requirement of additional hardware. They argued that the mobile application can detect if the user is sitting, travelling 
or walking. However, using GSM signals can fail sometimes due to instability of the network. The focus of Sawar et al.[13] was on adults. They developed a pedometer application using accelerometer sensor. Their objective was to encourage users to perform up to the level of physical activities recommended for them. They incorporated gamification technique to motivate and encourage users. Gamification technique is used for giving or reducing points to user to enable them stay for longer periods in the activity. Although its goal is similar, but the mobile application makes use of Google Fit API to recognize total number of steps taken by the user. They carried out experiments to determine the accuracy of the pedometer application using 5 participants. Each participant was asked to count his/her actual number of steps and compare it with the steps counted by the application; they argued that its performance is satisfactory with $86 \%$ accuracy. Hirzallah [5] also developed a game that makes players exercise while playing. Instead of sitting one place, the player is required to move around, thereby, keeping the player healthy and minimizing the chance of addiction. Consolve et al.[2] presented a prototype mobile based application to encourage physical activities by sharing movement details with friends. They used accelerometer sensor integrated in mobile phones which interacts with the mobile application to count steps taken by users. Thirteen participants were recruited to evaluate the system; they argued that most participants were eager to know their performance and were motivated to achieve their goal quicker. Bielik et al.[1] also implemented mobile application with intention to encourage healthier lifestyle and motivate people to participate in steady physical activities. The mobile application collects data from different sensors such as GSM, Accelerometer and GPS in order to recognize and access physical activities. They claimed that their system is effective and sustainable, but, using those sensors involves high power consumption, thereby forcing users to always look for where to charge their mobile phones. To alleviate this challenge, they used GSM signal fluctuation analysis and information about available Wi-fi access points to detect when activity is going on in order to turn off sensor. This method also created extra task for the user. Khalil and Glal [7] developed a step counter application on sensor enabled Nokia N95 phone and written in Symbian $\mathrm{C}++$. They argued that the mobile application automatically deduce the number of steps taken by the user. Their aim was to present to the user a measure of his or her everyday activities, increase user's consciousness and understanding of the necessity of physical exercise. A smartphone application was implemented by Rajanna et al. [12]. This application provides physical activity reminder by using setting information such as location calendar events, personal preference, weather and time of the day. Their aim is to encourage users to carry out physical exercises after a prolonged sedentary lifestyle. This application utilized the power of accelerometer sensor embedded in android device, using decision tree based machine learning algorithm to classify the state of the user as either activity or sedentary. Pina et al. [11] also designed a system that detects sedentary behaviour. Their aim is to detect when users are inactive for long periods of time and prompt them to take a break and walk around.

\section{SYSTEM IMPLEMENTATION}

\subsection{System Architecture}

ActiveApp system is a mobile application which interacts with web applications to submit details of the user to database. The mobile application is compatible with android version 4.4 and above. The web application is developed using PHP scripting language to enable communication between the mobile application and MySQL database. MySQL database is used to store users' details and activity details. When the mobile app is installed on mobile phone, the IP address of the server will be supplied. The mobile phone connects default HTTP client. Then a web connection is established with the server and login information validated, the mobile app will be able to collect daily activity details of the participant and submit same to the server. The system architecture is illustrated in the Figure 1 below.

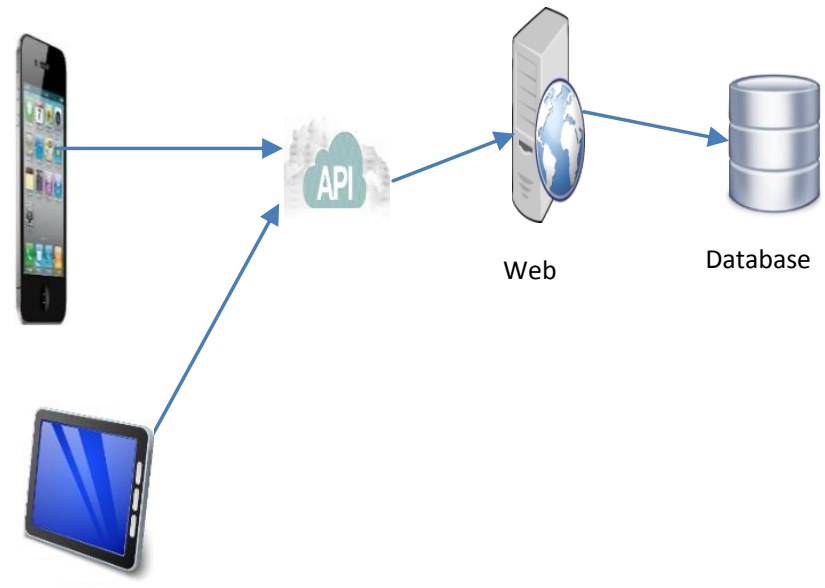

Figure1: ActiveApp system architecture

\subsection{System Description}

The android based application has user interfaces that interact with users. The registration page enables the user to supply his/her details which will be validated during login to be able to record activity details. When user is validated, he/she is taken to the main page to record his/her activity details. The main page contains interface which interact with mobile phone embedded sensor to display total steps taken by the user and the corresponding time taken by the user. The system is implemented in such a way that users' activity details-Steps and total time covered are submitted to the database at predetermined time interval. Figure 2, 3 and 4 below shows the system login page, registration page and the main page for activity tracking respectively. 


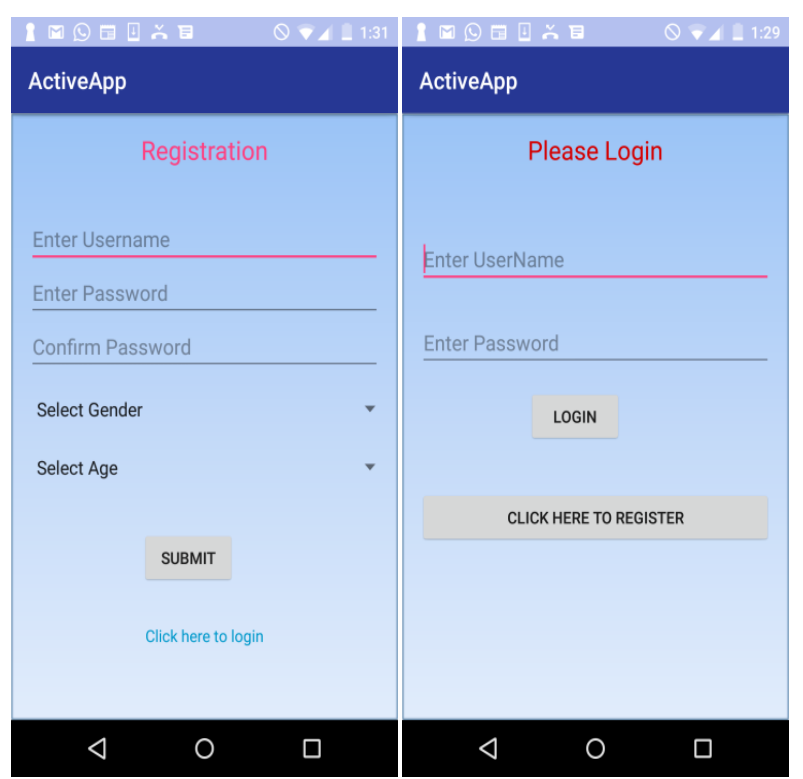

Figure 2: System login

Figure 3: System registration page

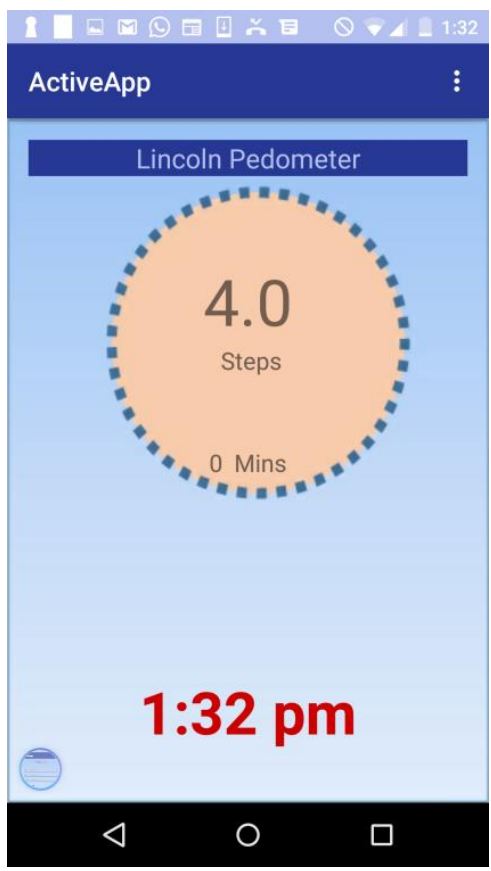

Figure4: Step count page

\section{PERFORMANCE EVALUATION}

To evaluate the developed mobile app, ten persons ( 5 males and 5 females) were recruited and asked to carry Nexus 5 phone running the app and walk around. Each person counted the actual steps taken and the steps recorded by the app. The table below shows the recorded steps from the mobile phone and the actual steps of each person.

Table 1: Summary of results obtained in evaluating ActiveApp

\begin{tabular}{|l|l|l|l|}
\hline Participant & $\begin{array}{l}\text { Actual } \\
\text { Steps }\end{array}$ & $\begin{array}{l}\text { Recorded } \\
\text { Steps }\end{array}$ & Accuracy \\
\hline 1 & 50 & 48 & $96 \%$ \\
\hline 2 & 400 & 380 & $95 \%$ \\
\hline
\end{tabular}

\begin{tabular}{|l|l|l|l|}
\hline 3 & 150 & 142 & $95 \%$ \\
\hline 4 & 200 & 184 & $92 \%$ \\
\hline 5 & 500 & 475 & $95 \%$ \\
\hline 6 & 300 & 285 & $95 \%$ \\
\hline 7 & 100 & 93 & $93 \%$ \\
\hline 8 & 450 & 430 & $96 \%$ \\
\hline 9 & 350 & 330 & $94 \%$ \\
\hline 10 & 250 & 225 & $90 \%$ \\
\hline
\end{tabular}

From Table 1 above, ActiveApp recorded average accuracy of 94\% and could potentially be used to record daily steps count of users. Installing this app will motivate users to engage in physical activities, since they will be happy to record high levels of step counts each day.

\section{CONCLUSION}

The healthcare benefits associated with regular monitoring of physical activities cannot be overemphasized. It positions individuals in a state of fitness. Mobile application is transforming healthcare the same way it has changed the way people live, work and interact with each other. There is no doubt that mobile healthcare applications will massively facilitate a future in which patients will be given the appropriate tools to manage their health. In this paper, we have presented smartphone-based step tracking system (ActiveApp). The system tracks the daily steps count of users carrying mobile phones. The experimental evaluation shows that the system can effectively record and track steps of users in real time. In future, we will implement real time human activity recognition system using smartphone sensor. The system will be able to recognize a particular activity of the user.

\section{REFERENCES}

[1] Bielik, P., Tomlein, M., Krátky, P., Mitrík, Š., Barla, M., \& Bieliková, M. (2012). Move2Play: an innovative approach to encouraging people to be more physically active. In Proceedings of the 2nd ACM SIGHIT international health informatics symposium (pp. 61-70). ACM.

[2] Consolvo, S., Everitt, K., Smith, I., \& Landay, J. A. (20061). Design requirements for technologies that encourage physical activity. In Proceedings of the SIGCHI conference on Human Factors in computing systems (pp. 457-466). ACM.

[3] Gjoreski, M., Gjoreski, H., Luštrek, M., \& Gams, M. (2016). How accurately can your wrist device recognize daily activities and detect falls?. Sensors, 16(6), 800.

[4] Hendeby, G., Bleser, G., Lamprinos, I., \& Stricker, D. (2010). Healthy aging using physical activity monitoring. German Research, 122, 67663.Elserver Clinical solutions (2015); Mobile Applications and the Future of Healthcare, White Paper

[5] Smallman G. (2012); The Benefit of apps in Healthcare, The Guardian

[6] Hirzallah, N. (2013). A simple exercise-to-play proposal that would reduce games addiction and keep players 
healthy. International Journal of Advanced Computer Science and Applications (IJACSA), 4(2).

[7] Kwapisz, J. R., Weiss, G. M., \& Moore, S. A. (2010). Activity Recognition using Cell Phone Accelerometers.

[8] Khalil, A., \& Glal, S. (2009). StepUp: A step counter mobile application to promote healthy lifestyle. In Current Trends in Information Technology (CTIT), 2009 International Conference on the (pp. 1-5). IEEE.

[9] Lin, Y., Jessurun, J., De Vries, B., \& Timmermans, H. (2011). Motivate: Towards context-aware recommendation mobile system for healthy living. In Pervasive Computing Technologies for Healthcare (PervasiveHealth), 2011 5th International Conference on (pp. 250-253). IEEE.

[10] Maitland, J., Sherwood, S., Barkhuus, L., Anderson, I., Hall, M., Brown, B., \& Muller, H. (2006). Increasing the awareness of daily activity levels with pervasive computing. In Pervasive Health Conference and Workshops, 2006 (pp. 1-9). IEEE.
[11] Pina, L. R., Ramirez, E., \& Griswold, W. G. (2012). Fitbit+: A behavior-based intervention system to reduce sedentary behavior. In Pervasive Computing Technologies for Healthcare (PervasiveHealth), 2012 6th International Conference on (pp. 175-178). IEEE.

[12] Rajanna, V., Lara-Garduno, R., Behera, D. J., Madanagopal, K., Goldberg, D., \& Hammond, T. (2014). Step up life: a context aware health assistant. In Proceedings of the Third ACM SIGSPATIAL International Workshop on the Use of GIS in Public Health (pp. 21-30). ACM.

[13] Sarwar, A., Mukhtar, H., Maqbool, M., \& Belaid, D. SmartFit: A Step Count Based Mobile Application for Engagement in Physical Activities. International Journal of Advanced Computer Science \& Applications, 1(6), 271-278.

[14] Van Dantzig, S., Geleijnse, G., \& van Halteren, A. T. (2013). Toward a persuasive mobile application to reduce sedentary behavior. Personal and ubiquitous computing, 17(6), 1237-1246. 